\title{
On a Min-Max Procedure for the Existence of a Positive Solution for Certain Scalar Field Equations in $\mathbb{R}^{N}$
}

Abbas Bahri and Yan Yan Li

\begin{abstract}
In this paper we mainly introduce a min-max procedure to prove the existence of positive solutions for certain semilinear elliptic equations in $\mathbb{R}^{N}$.
\end{abstract}

\section{Introduction}

In this paper, we investigate the existence of positive solutions for the following semilinear elliptic equation in $\mathbb{R}^{N}$ :

$$
\left\{\begin{array}{l}
-\Delta u+u-q(x)|u|^{p-1} u=0 \\
u \in H^{1}\left(\mathbb{R}^{N}\right)
\end{array}\right.
$$

where $1<p<\frac{N+2}{N-2}$, if $N \geqslant 3 ; 1<p<+\infty$, if $N=1,2$ and $q \in L^{\infty}\left(\mathbb{R}^{N}\right)$ satisfies the following hypotheses:

$$
q(x)>0 \text { for every } x \in \mathbb{R}^{N} .
$$


There exists a positive constant, denoted as $q_{\infty}$, such that

$$
\lim _{|x| \rightarrow \infty} q(x)=q_{\infty}
$$

and there exist some positive constants $C$ and $\delta$ such that, for $|x|$ large,

$$
q(x) \geqslant q_{\infty}-C \exp (-\delta|x|) .
$$

Under the hypotheses (2), (3) and (4), we are able to prove the existence of at least one positive solution to (1). However the main purpose of this paper is to derive the result through a min-max procedure, similar in spirit to [6], even though we have to replace (4) by a stronger hypothesis:

There exist some positive constants $C$ and $\delta$ such that, for $|x|$ large,

$$
q(x) \geqslant q_{\infty}-C \exp (-(2+\delta)|x|) .
$$

Without loss of generality we can assume that $\delta<p-1$.

The existence of solutions of semilinear elliptic equations in $\mathbb{R}^{N}$ have been investigated, among others, in [3], [8], [13], [14], [5], [2], [18], [19], [11].

Weiyue Ding and Wei-Ming Ni established in [8] that (1) has at least one positive solution if $q(x)>0$ is radially symmetric and bounded, for large $|x|$, by $|x|^{l}$ where $l$ satisfies $0<l<\frac{(N-1)(p-1)}{2}$. On the other hand $\mathrm{Yi} \operatorname{Li}([12])$ has proved that for $N \geqslant 3$ (1) has no positive solution if $q(x) \geqslant 0, q \in C^{0,1}\left(\mathbb{R}^{N}\right)$ is radially symmetric and $q(x)|x|^{-(N-1)(p-1) / 2}$ is nondecreasing in $|x|$. However, very little is known for the existence of nontrivial solution of (1) if $q(x)$ is not radially symmetric. In [8] [13], variational methods are used to prove the existence of a positive solution of (1) under various hypotheses, which ensure the existence of the global minimum of the functional associated with (1). Therefore when the minimum is not achieved the existence problem is left open. It is proved by A. Bahri and P. L. Lions in [2] that if we consider the problem on $\mathbb{R}^{N} \backslash \Omega$ instead of $\mathbb{R}^{N}$, where $\Omega$ is any smooth bounded open set, then the existence of positive solution can be established even when the minimum of the functional is not achieved. There the topology of $\mathbb{R}^{N} \backslash \Omega$ has been used. Our present situation is different since $\mathbb{R}^{N}$ is a contractable set. Therefore the technique developed in [2] cannot be applied directly. However, with the observation we have here, we can modify the argument of [2] to prove the existence of positive solution without using the topology of the domain.

In [5] and [11] the existence of multiple solutions has been studied under various hypotheses on $q(x)$. 
On a Min-Max Procedure for the Existence of a Positive Solution 3

\section{Preliminaries}

We first introduce some notations. Let

$$
\langle u, v\rangle=\int_{\mathbb{R}^{N}} \nabla u \nabla v+u v
$$

denote the inner product of $H^{1}\left(\mathbb{R}^{N}\right)$ and

$$
\begin{gathered}
\|u\|=\langle u, u\rangle^{1 / 2} \\
\Sigma=\left\{u \in H^{1}\left(\mathbb{R}^{N}\right):\|u\|=1\right\} \\
\Sigma^{+}=\left\{u \in \Sigma: u \geqslant 0 \text { almost everywhere in } \mathbb{R}^{N}\right\} .
\end{gathered}
$$

Let

$$
\begin{aligned}
J(u) & =\frac{\int_{\mathbb{R}^{N}}|\nabla u|^{2}+u^{2}}{\left(\int_{\mathbb{R}^{N}} q(x)|u|^{p+1}\right)^{2 /(p+1)}} \\
J_{\infty}(u) & =\frac{\int_{\mathbb{R}^{N}}|\nabla u|^{2}+u^{2}}{\left(\int_{\mathbb{R}^{N}} q_{\infty}|u|^{p+1}\right)^{2 /(p+1)}} \\
S_{1} & =\inf _{u \in H^{1}\left(\mathbb{R}^{N}\right) \backslash\{0\}} J_{\infty}(u) \\
S_{m} & =m^{(p-1) /(p+1)} S_{1}, \quad m=2,3,4, \ldots
\end{aligned}
$$

It is well known that $S_{1}$ is actually achieved by some radially symmetric positive smooth function $\omega$, which satisfies the following equation

$$
\left\{\begin{array}{l}
-\Delta \omega+\omega-q_{\infty} \omega^{p}=0, \\
\omega \in H^{1}\left(\mathbb{R}^{N}\right) .
\end{array}\right.
$$

It has also been proved by Kwong ([10]) recently that the positive solution of (6) is actually unique up to translations. Furthermore we know exactly how $\omega$ behaves at $+\infty$.

Theorem 1.1. Let $q(x) \in L^{\infty}\left(\mathbb{R}^{N}\right)$ satisfy (2), (3) and (5). Then (1) has a positive solution in $H^{1}\left(\mathbb{R}^{N}\right)$ for $1<p<(N+2) /(N-2)$, if $N \geqslant 3 ; 1<p<$ $+\infty$, if $N=1,2$.

Remark 1.1. The regularity of the solution found in Theorem 1.1 follows from standard elliptic theory.

Proposition 1.1. Let $\omega$ be the positive solution of (6), then $\omega \in C^{\infty}\left(\mathbb{R}^{N}\right)$ and is radially symmetric after suitable translation, namely, $\omega=\omega(|x|)$. Further- 
more, there exists some positive constant $c>0$, such that,

$$
\begin{array}{cccc}
\omega(|x|)|x|^{(N-1) / 2} \exp (|x|) \rightarrow c & \text { as } & |x| \rightarrow \infty \\
\omega^{\prime}(|x|)|x|^{(N-1) / 2} \exp (|x|) \rightarrow-c & \text { as } & |x| \rightarrow \infty
\end{array}
$$

The proof of the above proposition can be found in [2] and the references there.

Proposition 1.2. Let $\varphi \in C\left(\mathbb{R}^{N}\right) \cap L^{\infty}\left(\mathbb{R}^{N}\right), \psi \in C\left(\mathbb{R}^{N}\right)$ be radially symmetric and satisfy for some $\alpha \geqslant 0, \beta \geqslant 0, \gamma \in \mathbb{R}$

$$
\begin{gathered}
\varphi(x) \exp (\alpha|x|)|x|^{\beta} \rightarrow \gamma \text { as }|x| \rightarrow \infty, \\
\int_{\mathbb{R}^{N}}|\psi(x)| \exp (\alpha|x|)\left(1+|x|^{\beta}\right)<\infty .
\end{gathered}
$$

Then

$\left(\int_{\mathbb{R}^{N}} \varphi(x+y) \psi(x) d x\right) \exp (\alpha|y|)|y|^{\beta} \rightarrow \gamma \int_{\mathbb{R}^{N}} \psi(x) \exp \left(-\alpha x_{1}\right) d x$ as $|y| \rightarrow \infty$.

PRoof. Since $\varphi, \psi$ are radially symmetric, we only need to obtain the limit for $y=(|y|, 0, \ldots, 0),|y| \rightarrow+\infty$.

In the following we then assume that $y=(|y|, 0, \ldots, 0)$. We first identify the pointwise limit of the integrand.

$$
\begin{aligned}
& \varphi(x+y) \psi(x) \exp (\alpha|y|)|y|^{\beta} \\
& =\psi(x) \varphi(x+y) \exp (\alpha|x+y|)|x+y|^{\beta} \exp (-\alpha|x+y|) \exp (\alpha|y|)|x+y|^{-\beta}|y|^{\beta}
\end{aligned}
$$

The limit (9) implies that

$$
\varphi(x+y) \exp (\alpha|x+y|)|x+y|^{\beta} \rightarrow \gamma
$$

pointwise as $|y| \rightarrow+\infty$. On the other hand,

$$
\lim _{|y| \rightarrow+\infty}|x+y|^{-\beta}|y|^{\beta}=1
$$

is obvious.

Using the fact that $y=(|y|, 0, \ldots, 0)$, we have

$$
\begin{aligned}
\exp (-\alpha|x+y|) \exp (\alpha|y|) & =\exp \left(-\alpha\left(|x|^{2}+2 x \cdot y+|y|^{2}\right)^{1 / 2}\right) \exp (\alpha|y|) \\
& =\exp \left(-\alpha|y|\left(1+2 \frac{x \cdot y}{|y|}+\frac{|x|^{2}}{|y|^{2}}\right)^{1 / 2}\right) \exp (\alpha|y|) \\
& =\exp \left(-\alpha|y|-\alpha x_{1}+O\left(\frac{1}{|y|}\right)\right) \exp (\alpha|y|) .
\end{aligned}
$$


Therefore

$$
\lim _{\substack{|y| \rightarrow+\infty \\ y=(|y|, 0, \ldots, 0)}} \exp (-\alpha|x+y|) \exp (\alpha|y|)=\exp \left(-\alpha x_{1}\right) .
$$

Putting the above calculations together, we obtain that

$$
\lim _{\substack{|y| \rightarrow+\infty \\ y=(|y|, 0, \ldots, 0)}} \varphi(x+y) \psi(x) \exp (\alpha|y|)|y|^{\beta}=\gamma \psi(x) \exp \left(-\alpha x_{1}\right) .
$$

It follows from (9) that

$$
\begin{aligned}
\left.|\varphi(x+y) \psi(x) \exp (\alpha|y|)| y\right|^{\beta} \mid & \\
& \leqslant C|\psi(x)| \exp (-\alpha|x+y|)(1+|x+y|)^{-\beta} \exp (\alpha|y|)|y|^{\beta} \\
& \leqslant C|\psi(x)| \exp (\alpha|x|)(1+|x+y|)^{-\beta}|y|^{\beta} .
\end{aligned}
$$

For $|y| \geqslant 2|x|$,

$$
\left.|\varphi(x+y) \psi(x) \exp (\alpha|y|)| y\right|^{\beta}|\leqslant C| \psi(x) \mid \exp (\alpha|x|) .
$$

For $|y| \leqslant 2|x|$,

$$
\left.\left.|\varphi(x+\ddot{y}) \psi(x) \exp (\alpha|y|)| y\right|^{\beta}|\leqslant C| \psi(x)|\exp (\alpha|x|)| x\right|^{\beta} .
$$

Now we can simply apply the Lebesgue dominated convergence theorem to conclude the proof of Proposition 1.2.

To apply a min-max method, we need to analyze where the functional $J$ satisfies certain compactness condition. This has been well known by now. In the following, we are going to state some compactness lemmas. For the proof, see [13], [14] and [2].

Definition 1.1. $\left.J\right|_{\Sigma}$ is said to satisfy Palais-Smale condition at $C^{\prime}$, if for any Palais-Smale sequence $\left\{u_{n}\right\} \in \Sigma$, namely, $\left\{u_{n}\right\} \in \Sigma, J\left(u_{n}\right) \rightarrow C^{\prime},\left.J\right|_{\Sigma} ^{\prime}\left(u_{n}\right) \rightarrow 0$ strongly in $H^{1}\left(\mathbb{R}^{N}\right)$, there exists a subsequence of $\left\{u_{n}\right\}$ which converges strongly in $H^{1}\left(\mathbb{R}^{N}\right)$.

Lemma. Let $\left\{u_{n}\right\} \in \Sigma$ be a Palais-Smale sequence, then there exists a subsequence (still denoted as $\left.\left\{u_{n}\right\}\right)$ for which the following holds: there exists an integer $m \geqslant 0$, sequences $x_{n}^{i}$ for $1 \leqslant i \leqslant m$, functions $\bar{u}, \omega_{i}$ for $1 \leqslant i \leqslant m$ such that

$$
\begin{aligned}
& \text { (11) } \quad-\Delta \bar{u}+\bar{u}=q(x)|\bar{u}|^{p-1} \bar{u} \text { in } \mathbb{R}^{N}, \quad \bar{u} \in H^{1}\left(\mathbb{R}^{N}\right) \\
& \text { (12) } \quad-\Delta \omega_{i}+\omega_{i}=q_{\infty}\left|\omega_{i}\right|^{p-1} \omega_{i} \text { in } \mathbb{R}^{N}, \quad \omega_{i} \in H^{1}\left(\mathbb{R}^{N}\right) \\
& \text { (13) }\left|x_{n}^{i}\right| \rightarrow \infty, \quad\left|x_{n}^{i}-x_{n}^{j}\right| \rightarrow \infty \text { as } n \rightarrow \infty \text { for } \quad l \leqslant i \neq j \leqslant m
\end{aligned}
$$




$$
\begin{gathered}
u_{n}-\frac{\bar{u}+\sum_{i=1}^{m} \omega_{i}\left(\cdot-x_{n}^{i}\right)}{\left\|\bar{u}+\sum_{i=1}^{m} \omega_{i}\left(\cdot-x_{n}^{i}\right)\right\|} \rightarrow 0 \text { strongly in } H^{1}\left(\mathbb{R}^{N}\right) \text { as } n \rightarrow \infty \\
J\left(u_{n}\right) \rightarrow \frac{\|\bar{u}\|^{2}+\sum_{i=1}^{m}\left\|\omega_{i}\right\|^{2}}{\left(\int_{\mathbb{R}^{N}} q|\bar{u}|^{p+1}+\sum_{i=1}^{m} \int_{\mathbb{R}^{N}} q_{\infty}\left|\omega_{i}\right|^{p+1}\right)^{\frac{2}{p+1}}} \text { as } n \rightarrow \infty
\end{gathered}
$$

where we agree that in the case $m=0$ the above holds without $\omega_{i}$ and $x_{n}^{i}$. In addition, if $u_{n} \geqslant 0$ then $\bar{u} \geqslant 0, \omega_{i} \geqslant 0$ for all $1 \leqslant i \leqslant m$. Therefore $\omega_{i} \equiv \omega$ for all $1 \leqslant i \leqslant m$ due to the uniqueness upto translation of positive solution of (6) (see [10]).

This result immediately implies that if $u_{n} \geqslant 0$ in the PS Lemma and (1) has no positive solution, then

$$
J\left(u_{n}\right) \rightarrow S_{m}
$$

where $m \geqslant 1$ is some integer.

We are going to use a contradiction argument for the existence of positive solution of (1), namely, we start with the assumption that (1) has no positive solution, then we know that (PS) condition fails only at levels $\left\{S_{m}\right\}$. Furthermore if we are able to construct some min-max value which is different from $\left\{S_{m}\right\}$, the standard deformation lemma will give rise to a positive solution to (1), hence a contradiction. The purpose of the next two sections is to construct such a min-max value which is strictly between $S_{1}$ and $S_{2}$.

\section{Energy Estimates}

The energy estimates given in this section play an important role for the proof of the existence result. These estimates have essentially been given in [2], while we do need a result which is slightly different from that in [2]. This kind of phenomenon have been used by C. Taubes ([17]), A. Bahri and J. M. Coron ([1]), A. Bahri and P. L. Lions ([2]).

We first state a result which will be used later.

Lemma 2.1. Let $p>1$ be any real number, then there exists some constant $C=C(p)$, such that, for any nonnegative real numbers $a, b$,

(17) $(a+b)^{p+1} \geqslant a^{p+1}+b^{p+1}+(p+1)\left(a^{p} b+a b^{p}\right)-C a^{(p+1) / 2} b^{(p+1) / 2}$

(In fact, if $p \geqslant 2$, we may take $C=C(p)=0$.) 
Proof. If $a=0$ or $b=0$, (17) is obvious. Otherwise we assume, without loss of generality, that $a \leqslant b, a b \neq 0$. Let $x=a / b$, then $0<x \leqslant 1$, (17) is equivalent to the following:

$$
(1+x)^{p+1} \geqslant 1+x^{p+1}+(p+1)\left(x+x^{p}\right)-C x^{(p+1) / 2} .
$$

For $x$ small, it follows from Taylor expansion that

$$
(1+x)^{p+1}=1+(p+1) x+\frac{p(p+1)}{2} x^{2}+o\left(x^{3}\right),
$$

therefore there exists some positive number $\delta_{0}=\delta_{0}(p)$ between 0 and 1, such that, (18) holds for $0<x \leqslant \delta_{0}, C \geqslant p+1$. For $\delta_{0} \leqslant x \leqslant 1$, we can choose $C=C(p)$ large enough to guarentee (18).

Lemma 2.2. Under the hypotheses of Theorem 1.1, there exists a large number $R_{0}$, such that, for any $R \geqslant R_{0},\left|x_{1}\right| \geqslant R,\left|x_{2}\right| \geqslant R, \sqrt{R} \leqslant\left|x_{1}-x_{2}\right| \leqslant$ $(2+1 / 2 \sqrt{R}) \min \left\{\left|x_{1}\right|,\left|x_{2}\right|\right\}$, we have

$$
J\left(t u_{1}+(1-t) u_{2}\right)<S_{2}
$$

where $0 \leqslant t \leqslant 1$ and $u_{i}=\omega\left(\cdot-x_{i}\right)$.

Proof. Let us first prove (19) for $t=1 / 2$, namely,

$$
J\left(u_{1}+u_{2}\right)<S_{2} .
$$

Let

$$
A=\|\omega\|^{2}
$$

then from (6) we know that

$$
A=\int q_{\infty} \omega^{p+1} .
$$

The following computation holds for large $R$.

$$
\begin{aligned}
J\left(u_{1}+u_{2}\right) & =\frac{\left\|u_{1}+u_{2}\right\|^{2}}{\left(\int q\left(u_{1}+u_{2}\right)^{p+1}\right)^{2 /(p+1)}} \\
& =\frac{\left\|u_{1}\right\|^{2}+\left\|u_{2}\right\|^{2}+2\left\langle u_{1}, u_{2}\right\rangle}{\left(\int q_{\infty}\left(u_{1}+u_{2}\right)^{p+1}+\int\left(q-q_{\infty}\right)\left(u_{1}+u_{2}\right)^{p+1}\right)^{2 /(p+1)}} \\
& \leqslant \frac{2 A+2\left\langle u_{1}, u_{2}\right\rangle}{\left(\int q_{\infty}\left(u_{1}+u_{2}\right)^{p+1}-\int\left(q_{\infty}-q\right)^{+}\left(u_{1}+u_{2}\right)^{p+1}\right)^{2 /(p+1)}}
\end{aligned}
$$


We first estimate $\int u_{1}^{p} u_{2}$. In the following $C$ will denote some positive constant independent of $R$.

$$
\begin{aligned}
\int u_{1}^{p} u_{2} & =\int \omega\left(\left|x-x_{1}\right|\right)^{p} \omega\left(\left|x-x_{2}\right|\right) d x \\
& \geqslant \int_{\left|x-x_{1}\right| \leq 1} \omega\left(\left|x-x_{1}\right|\right)^{p} \omega\left(\left|x-x_{2}\right|\right) d x \\
& \geqslant \frac{1}{C} \int_{\left|x-x_{1}\right| \leq 1} \omega\left(\left|x-x_{2}\right|\right) d x \\
& \geqslant \frac{1}{C} \omega\left(\left|x_{1}-x_{2}\right|+1\right) \\
& \geqslant \frac{1}{C}\left|x_{1}-x_{2}\right|^{(1-N) / 2} \exp \left(-\left|x_{1}-x_{2}\right|\right)
\end{aligned}
$$

The last inequality follows from (7).

Secondly we estimate $\int u_{1}^{(p+1) / 2} u_{2}^{(p+1) / 2}$.

$$
\begin{aligned}
\int u_{1}^{(p+1) / 2} u_{2}^{(p+1) / 2} & =\int \omega\left(\left|x-x_{1}\right|\right)^{(p+1) / 2} \omega\left(\left|x-x_{2}\right|\right)^{(p+1) / 2} d x \\
& \leqslant o(1)\left|x_{1}-x_{2}\right|^{(1-N) / 2} \exp \left(-\left|x_{1}-x_{2}\right|\right) \\
& \leqslant o(1) \int u_{1}^{p} u_{2}
\end{aligned}
$$

where (17) and Proposition 1.2 have been used.

Here and in the sequel, $o(1) \rightarrow 0$ as $R$ goes to $\infty$.

Thirdly we estimate $\int\left(q_{\infty}-q\right)^{+}\left(u_{1}+u_{2}\right)^{p+1}$. Use (5), (7) and Proposition 1.2 , we have

$$
\begin{aligned}
\int\left(q_{\infty}-q\right)^{+}\left(u_{1}+u_{2}\right)^{p+1} & \leqslant C \sum_{i=1}^{2} \int\left(q_{\infty}-q\right)^{+} u_{i}^{p+1} \\
& \leqslant C \sum_{i=1}^{2} \int \exp (-(2+\delta)|x|) \omega\left(\left|x-x_{i}\right|\right)^{p+1} d x \\
& \leqslant C \sum_{i=1}^{2} \exp \left(-\frac{2+\delta}{2}\left|x_{i}\right|\right) \\
& \leqslant C \sum_{i=1}^{2} \exp \left(-\frac{2+\delta}{2} \frac{\left|x_{1}-x_{2}\right|}{2+\frac{1}{2 \sqrt{R}}}\right) \\
& =o(1)\left|x_{1}-x_{2}\right|^{(1-N) / 2} \exp \left(-\left|x_{1}-x_{2}\right|\right)=o(1) \int u_{1}^{p} u_{2}
\end{aligned}
$$


where we have used the hypothesis

$$
\sqrt{R} \leqslant\left|x_{1}-x_{2}\right| \leqslant\left(2+\frac{1}{2 \sqrt{R}}\right) \min \left\{\left|x_{1}\right|,\left|x_{2}\right|\right\} .
$$

According to Lemma 2.1 and the above estimates, we deduce that

$$
\begin{aligned}
\int q_{\infty}\left(u_{1}+u_{2}\right)^{p+1} & \\
& \geqslant \int q_{\infty}\left(u_{1}^{p+1}+u_{2}^{p+1}\right)+(p+1) \int q_{\infty}\left(u_{1}^{p} u_{2}+u_{1} u_{2}^{p}\right)-C \int u_{1}^{(p+1) / 2} u_{2}^{(p+1) / 2} \\
& =2 A+(2 p+2)\left\langle u_{1}, u_{2}\right\rangle-C \int u_{1}^{(p+1) / 2} u_{2}^{(p+1) / 2} \\
& \geqslant 2 A+(2 p+2-o(1))\left\langle u_{1}, u_{2}\right\rangle
\end{aligned}
$$

where we have used the fact that

$$
\int q_{\infty} u_{1}^{p} u_{2}=\int q_{\infty} u_{2}^{p} u_{1}=\left\langle u_{1}, u_{2}\right\rangle,
$$

which follows from (6).

Therefore

$$
\begin{aligned}
J\left(u_{1}+u_{2}\right) & \leqslant \frac{2 A+2\left\langle u_{1}, u_{2}\right\rangle}{\left(\int q_{\infty}\left(u_{1}+u_{2}\right)^{p+1}-\int\left(q_{\infty}-q\right)^{+}\left(u_{1}+u_{2}\right)^{p+1}\right)^{2 /(p+1)}} \\
& \leqslant \frac{2 A+2\left\langle u_{1}, u_{2}\right\rangle}{\left(2 A+(2 p+2-o(1))\left\langle u_{1}, u_{2}\right\rangle\right)^{2 / p+1)}} \\
& =S_{2} \frac{1+\frac{1}{A}\left\langle u_{1}, u_{2}\right\rangle}{\left(1+\frac{p+1-o(1)}{A}\left\langle u_{1}, u_{2}\right\rangle\right)^{2 /(p+1)}} \\
& =S_{2} \frac{1+\frac{1}{A}\left\langle u_{1}, u_{2}\right\rangle}{1+\frac{2 p+2-o(1)}{(p+1) A}\left\langle u_{1}, u_{2}\right\rangle}
\end{aligned}
$$

Notice that $\frac{1}{A}<\frac{2 p+2-o(1)}{(p+1) A}$ for $R$ large, we obtain (20) from the above estimates. Next we are going to prove (19).

Let

$$
\left\{\begin{array}{l}
v_{1}=t u_{1} \\
v_{2}=(1-t) u_{2}
\end{array}\right.
$$

where $0 \leqslant t \leqslant 1$. 


$$
\begin{aligned}
\left\|v_{1}+v_{2}\right\|^{2} & =\left\|v_{1}\right\|^{2}+\left\|v_{2}\right\|^{2}+2\left\langle v_{1}, v_{2}\right\rangle \\
& =t^{2}\left\|u_{1}\right\|^{2}+(1-t)^{2}\left\|u_{2}\right\|^{2}+2 t(1-t)\left\langle u_{1}, u_{2}\right\rangle \\
& =\left(t^{2}+(1-t)^{2}\right) A+2 t(1-t)\left\langle u_{1}, u_{2}\right\rangle \\
\int q\left|v_{1}+v_{2}\right|^{p+1} & \geqslant \int q_{\infty}\left|v_{1}+v_{2}\right|^{p+1}-\int\left(q_{\infty}-q\right)^{+}\left|v_{1}+v_{2}\right|^{p+1} .
\end{aligned}
$$

When $t$ or $1-t$ tends to zero, $v_{1}+v_{2}$ tends to $u_{2}$ or $u_{1}$ and $J\left(v_{1}+v_{2}\right)$ consequently converges to $S_{1}$. Therefore there exists some small constant $\delta^{\prime}>0$, such that, for any $\min \{t, 1-t\} \leqslant \delta^{\prime},(20)$ holds. Notice that $\delta^{\prime}>0$ is independent of $R$ large. In the following we always assume that $\min \{t, 1-t\} \geqslant \delta^{\prime}$.

Arguing as before, we have,

$$
\begin{aligned}
\int q_{\infty} \mid v_{1}+ & \left.v_{2}\right|^{p+1} \\
\geqslant & \left(t^{p+1}+(1-t)^{p+1}\right) A+(p+1)\left(t^{p}(1-t)+t(1-t)^{p}\right)\left\langle u_{1}, u_{2}\right\rangle \\
& -C \int u_{1}^{2 /(p+1)} u_{2}^{2(p+1)} \\
\geqslant & \left(t^{p+1}+(1-t)^{p+1}\right) A+(p+1)\left(t^{p}(1-t)+t(1-t)^{p}-o(1)\right)\left\langle u_{1}, u_{2}\right\rangle .
\end{aligned}
$$

We have derived before that

$$
\int\left(q_{\infty}-q\right)^{+}\left(u_{1}+u_{2}\right)^{p+1}=o(1)\left\langle u_{1}, u_{2}\right\rangle .
$$

Notice that $\min \{t, 1-t\} \geqslant \delta^{\prime}$, we have

$$
\int\left(q_{\infty}-q\right)^{+}\left|v_{1}+v_{2}\right|^{p+1} \leqslant o(1)\left(t^{p}(1-t)+t(1-t)^{p}\right)\left\langle u_{1}, u_{2}\right\rangle .
$$

Therefore

$$
\begin{aligned}
& J\left(v_{1}+v_{2}\right) \\
& \leqslant \frac{\left(t^{2}+(1-t)^{2}\right) A+2 t(1-t)\left\langle u_{1}, u_{2}\right\rangle}{\left\{\left(t^{P+1}+(1-t)^{p+1}\right) A+(p+1)\left(t^{p}(1-t)+t(1-t)^{p}-o(1)\right)\left\langle u_{1}, u_{2}\right\rangle\right\}^{2 /(p+1)}} \\
& \leqslant \frac{t^{2}+(1-t)^{2}}{\left(t^{p+1}+(1-t)^{p+1}\right)^{2 /(p+1)}} \frac{A}{A^{2 /(p+1)}} \\
& \quad \times \frac{1+\frac{2 t(1-t)}{\left(t^{2}+(1-t)^{2}\right) A}\left\langle u_{1}, u_{2}\right\rangle}{1+\left(\frac{2\left(t^{p}(1-t)+t(1-t)^{p}\right)}{\left(t^{p+1}+(1-t)^{p+1}\right) A}-o(1)\right)\left\langle u_{1}, u_{2}\right\rangle} .
\end{aligned}
$$

Since

$$
\frac{A}{A^{2 /(p+1)}}=S_{1}=S_{2} \frac{2^{2 /(p+1)}}{2},
$$


we have

$$
\begin{aligned}
J\left(v_{1}+v_{2}\right) \leqslant S_{2} \frac{2^{2 /(p+1)}}{2} \frac{t^{2}+(1-t)^{2}}{\left(t^{p+1}+(1-t)^{p+1}\right)^{2 /(p+1)}} & \frac{1+\frac{2 t(1-t)}{\left(t^{2}+(1-t)^{2}\right) A}\left\langle u_{1}, u_{2}\right\rangle}{} \\
& \frac{1+\left(\frac{2\left(t^{p}(1-t)+t(1-t)^{p}\right)}{\left(t^{p+1}+(1-t)^{p+1}\right) A}-o(1)\right)\left\langle u_{1}, u_{2}\right\rangle}{1+} .
\end{aligned}
$$

Notice that we have the following inequalities:

$$
\begin{aligned}
& \frac{2^{2 /(p+1)}}{2} \frac{t^{2}+(1-t)^{2}}{\left(t^{p+1}+(1-t)^{p+1}\right)^{2 /(p+1)}} \leqslant 1 \text { for } 0 \leqslant t \leqslant 1 \\
& \frac{t(1-t)}{t^{2}+(1-t)^{2}}<\frac{t^{p}(1-t)+t(1-t)^{p}}{t^{p+1}+(1-t)^{p+1}} \text { for } 0<t<1
\end{aligned}
$$

(21) follows from the convexity of the function $x \mapsto x^{(p+1) / 2}, x \geqslant 0$ and (22) is elementary.

Since $t$ and $1-t$ are bounded away from zero, we have

$$
1+\frac{2 t(1-t)}{\left(t^{2}+(1-t)^{2}\right) A}\left\langle u_{1}, u_{2}\right\rangle<1+\left(\frac{2\left(t^{p}(1-t)+t(1-t)^{p}\right)}{\left(t^{p+1}+(1-t)^{p+1}\right) A}-o(1)\right)\left\langle u_{1}, u_{2}\right\rangle
$$

for $R$ sufficiently large.

Finally (19) follows from the above estimates.

\section{The min-max Procedure}

Let us define a map from $\Sigma$ to the unit ball of $\mathbb{R}^{N}$

$$
m(u)=\frac{1}{\|u\|_{p+1}^{p+1}} \int \frac{x}{|x|}|u|^{p+1} d x
$$

where $\|u\|_{p+1}$ denotes the $L^{p+1}\left(\mathbb{R}^{N}\right)$ norm of $u$.

Clearly, $m$ is continuous from $\Sigma$ to $\mathbb{R}^{N}$ and $|m(u)|<1$.

Let

$$
I_{a}=\inf _{\substack{m(u)=a \\ u \in \Sigma}} \frac{\|u\|^{2}}{\left(\int q|u|^{p+1}\right)^{2 /(p+1)}}
$$

where $a \in \mathbb{R}^{N}$ and $|a|<1$. 
It is well known that (1) has a positive solution, which is actually a constant multiple of the global minimum of the functional $J$, if $\inf _{u \in \Sigma} J(u)<S_{1}$. (See [13].)

In the following we always consider the case when $\inf _{u \in \Sigma} J(u) \geqslant S_{1}$.

If for some $a \in \mathbb{R}^{N},|a|<1, I_{a}=S_{1}$, then there exists some $u \in \Sigma^{+}$, $m(u)=a$, such that $J(u)=S_{1}$. This follows from the concentration-compactness principle developed in [13]. In this case we will also obtain a solution of (1) which is a constant multiple of the global minimum of $J$. See [13] for details.

There is only one possibility left, namely,

$$
I_{a}>S_{1} \text { for every } a \in \mathbb{R}^{N}, \quad|a|<1 .
$$

Fix any $a \in \mathbb{R}^{N},|a|<1$, since $I_{a}>S_{1}$, there exists some positive constant $R_{1}$, such that,

$$
J(\omega(\cdot-y))<\frac{1}{2}\left(I_{a}+S_{1}\right)<I_{a} \quad \text { for every } \quad y \in \mathbb{R}^{N}, \quad|y| \geqslant R_{1} .
$$

Let $R>\max \left\{R_{0}, R_{1}\right\}$ be very large. (In the following $R$ is always supposed to be very large), and

$$
\begin{gathered}
B_{R}(0)=\left\{x \in \mathbb{R}^{N}:|x|<R\right\} \\
\bar{x}_{2}=(0, \ldots, 0, R-\sqrt{R})
\end{gathered}
$$

We define a map $h_{0}$ from $\partial B_{R}(0)$ to $\Sigma^{+}$by

$$
h_{0}\left(x_{1}\right)=\frac{\omega\left(\cdot-x_{1}\right)}{\left\|\omega\left(\cdot-x_{1}\right)\right\|}
$$

where $x_{1} \in \partial B_{R}(0)$.

Since $R>R_{1}$, we have

$$
J\left(h_{0}\left(x_{1}\right)\right)<\frac{1}{2}\left(I_{a}+S_{1}\right)<I_{a} \text { for } x_{1} \in \partial B_{R}(0) .
$$

We define another map $h^{*}$ from $B_{R}(0)$ to $\Sigma^{+}$by

$$
h^{*}\left(t x_{1}+(1-t) \bar{x}_{2}\right)=\frac{t \omega\left(\cdot-x_{1}\right)+(1-t) \omega\left(\cdot-\bar{x}_{2}\right)}{\left\|t \omega\left(\cdot-x_{1}\right)+(1-t) \omega\left(\cdot-\bar{x}_{2}\right)\right\|}
$$

where $0 \leqslant t \leqslant 1, x_{1} \in \partial B_{R}(0)$. It is clear that $\left.h^{*}\right|_{\partial B_{R}(0)}=h_{0}$.

It follows from Lemma 2.2 that

$$
J\left(h^{*}(y)\right)<S_{2} \text { for all } y \in B_{R}(0) .
$$


We next define some min-max value. Let

$$
\Gamma=\left\{h: B_{R}(0) \rightarrow \Sigma^{+}: h \text { is continuous, }\left.h\right|_{\partial B_{R}(0)}=h_{0}\right\}
$$

and

$$
c_{0}=\inf _{h \in \Gamma} \max _{y \in B_{R}(0)} J(h(y))
$$

We will prove that

$$
S_{1}<I_{a} \leqslant c_{0}<S_{2}
$$

(25) implies that $c_{0}<S_{2}$.

Consider the map

$$
m \circ h: \quad B_{R}(0) \rightarrow R^{N}
$$

for $h \in \Gamma$.

It is quite obvious that

$$
\lim _{R \rightarrow+\infty} m \circ h_{0}\left(x_{1}\right)=\frac{x_{1}}{\left\|x_{1}\right\|} \text { uniformly for } \quad x_{1} \in \partial B_{R}(0) .
$$

By degree theory, for $R$ large enough, we have

$$
\operatorname{deg}\left(m \circ h, B_{R}(0), a\right)=1 .
$$

In particular there exists some $y \in B_{R}(0)$, such that, $m \circ h(y)=a$, hence $c_{0} \geqslant I_{a}>S_{1}$.

The PS Lemma in Section 1 guarentees that $\left.J\right|_{\Sigma+}$ satisfies PS condition at $c_{0}$ if (1) has no solution. Then by using some standard deformation argument and the maximum principle (see [16], [2]), $c_{0}$ is a critical value of $\left.J\right|_{\Sigma}$ with some corresponding critical point $u>0$, i.e., $\left.J\right|_{\Sigma} ^{\prime}(u)=0$. By scaling $u$ we obtain a positive solution of (1). Therefore in any case, (1) has a positive solution. The proof of Theorem 1.1 has been completed.

Remark 3.1. Under the hypotheses of Theorem 1.1, there exists a positive solution $u$ with

$$
J(u)<S_{2} .
$$

Remark 3.2. If we repace $\mathbb{R}^{N}$ by $\mathbb{R}^{N} \backslash \bar{\Omega}$ where $\Omega$ is any bounded smooth open set of $\mathbb{R}^{N}$, the proof still holds after simple modification.

With the observation in this paper, it is not difficult to see that we can modify the arguments in [2] to prove the following result. 
Theorem 3.1. Suppose that $q(x) \in L^{\infty}\left(\mathbb{R}^{N}\right)$ satisfy (2), (3) and (4), then (1) has a positive solution in $H^{1}\left(\mathbb{R}^{N}\right)$ for $1<p<(N+2) /(N-2)$, if $N \geqslant 3 ; 1<p<$ $+\infty$, if $N=1,2$.

Acknowledgement. The authors want to express their sincere thanks to Professor Nirenberg for his useful comments and encouragement.

\section{References}

[1] Bahri, A. and Coron, J.M. On a nonlinear elliptic equation involving the critical Sobolev exponent: The effect of the topology of the domain. Comm. Pure Appl. Math. 41(1988), 253-294.

[2] Bahri, A. and Lions, P. L. On the existence of a positive solution of semilinear elliptic equations in unbounded domains. Preprint.

[3] Berestycki, H. and Lions, P. L. Nonlinear scalar field equations. Arch. Rational Mech. Anal., I, 82(1983), 313-346; II, 82(1983), 347-376.

[4] Brezis, H. and Nirenberg, L. Positive solutions of nonlinear elliptic equations involving critical Sobolev exponents. Comm. Pure Appl. Math. 36(1983), 437-477.

[5] Berestycki, H. and Taubes, C. In preparation.

[6] Coron, J. M. Topologie et cas limite des injections de Sobolev. C. R. Acad. Sc. Paris, I, 299(1984), 209-212.

[7] Ding, Weiyue On a conformally invariant equation on $\mathbb{R}^{N}$. Preprint.

[8] Ding, Weiyue and Ni, Wei-Ming On the existence of positive entire solutions of a semilinear elliptic equation. Arch. Rational Mech. Anal. 91(1986), 288-308.

[9] Esteban, M. J. and Lions, P. L. Existence and nonexistence results for semilinear elliptic problems in unbounded domains. Proc. Roy. Soc. Edim. 93(1981), 1-14.

[10] Kwong, M. K. Uniqueness of positive solutions of $\Delta u-u+u^{p}=0$ in $\mathbb{R}^{n}$. To appear on Arch. Rational Mech. Anal.

[11] Li, Y.Y. Existence of Multiple Solutions of Semilinear Elliptic Equations in $\mathbb{R}^{N}$. To appear in the «Proceeding of the Conference on Variational Problems», Paris, 1988.

[12] Li, Yi Remarks on a semilinear elliptic equation on $\mathbb{R}^{n}$. J. Diff. Eqn. 74(1988), 34-49.

[13] Lions, P. L. The concentration-compactness principle in the calculus of variations. The locally compact case. Ann. Inst. Henri. Poincare, Analyse non linéaire 1(1984), 102-145 and 223-283.

[14] Lions, P. L. On positive solutions of semilinear elliptic equations in unbounded domains. Preprint.

[15] Ni, Wei-Ming Some aspects of semilinear elliptic equations. Lecture notes, $\mathrm{Na}$ tional Tsing Hua University, Taiwan.

[16] Rabinowitz, P.H. Variational methods for nonlinear eigenvalue problems, Edicioni Cremonese, Roma (1974), 141-195.

[17] Taubes, C. Min-Max theory for the Yang-Mills-Higgs equations, Comm. Math. Phys. 97(1985), 473-540. 
On a Min-Max Procedure for the Existence of a Positive Solution 15

[18] Zhang, Dong. Positive Solutions of Nonlinear Scalar Field Equations Involving Critical Sobolev Exponent, Acta Math. Sinica, 3(1987), 27-37.

Recibido: 31 de octubre de 1989.

Abbas Bahri

Department of Mathematics

Rutgers University

New Brunswick NJ 08903

U.S.A.
Yan Yan $\mathrm{Li}^{*}$

Department of Mathematics

Princeton University

Princeton, NJ 08544

U.S.A.

* Partially supported by NSF grant DMS-8907849. 